\title{
Zur Analyse lokaler energiepolitischer Konflikte. Skizze eines Analysewerkzeugs
}

\author{
Sören Becker ${ }^{1}$ Andrea Bues ${ }^{1} \cdot$ Matthias Naumann $^{1}$
}

Eingegangen: 23. März 2015 / Angenommen: 12. Januar 2016 / Online publiziert: 1. Februar 2016

(C) Springer-Verlag Berlin Heidelberg 2016

\begin{abstract}
Zusammenfassung $\mathrm{Ob}$ die Transformation zu einer nachhaltigen Energieversorgung gelingt, wird maßgeblich auf der lokalen Ebene entschieden. Die vielen lokalen Energiewenden verlaufen jedoch nicht immer reibungslos, häufig kennzeichnen lokale Konflikte um Anlagen erneuerbarer Energien oder um neue Stromnetze deren Umsetzung. Die Frage nach ihrer öffentlichen Akzeptanz ist verbunden mit grundsätzlichen Überlegungen über die zugrunde liegenden Ziele des Einsatzes erneuerbarer Energien ebenso wie mit der Frage, wer am Ende von den neuen Infrastrukturen erneuerbarer Energien profitiert. Die Antworten darauf hängen wiederum vom Ergebnis komplexer Debatten und multilateraler Verhandlungen über das ,öffentliche Interesse" (Gemeinwohl) und von der Gewinnung neuer Organisationsformen der Energieversorgung ab. Der Bei-
\end{abstract}

Der Artikel entstand im Rahmen des Forschungsprojektes „Lösung von lokalen energiepolitischen Konflikten und Verwirklichung von Gemeinwohlzielen durch neue Organisationsformen im Energiebereich (EnerLOG)“, das aus Mitteln der Fördermaßnahme „Umwelt- und gesellschaftsverträgliche Transformation des Energiesystems" vom Bundesministerium für Bildung und Forschung (BMBF) gefördert wurde (Förderkennzeichen: 01UN1207B). Dem Beitrag liegt das Working Paper „Die Analyse lokaler energiepolitischer Konflikte und das Entstehen neuer Organisationsformen. Theoretische Zugänge und aktuelle Herausforderungen“" zugrunde (Becker/Bues/Naumann 2014).

Matthias Naumann

Matthias.Naumann@leibniz-irs.de

Sören Becker

Soeren.Becker@leibniz-irs.de

Andrea Bues

Andrea.Bues@1eibniz-irs.de

Leibniz-Institut für Raumbezogene Sozialforschung (IRS),

Flakenstraße 29-31, 15537 Erkner, Deutschland trag stellt ein Analysewerkzeug vor, das lokale energiepolitische Konflikte mit Blick auf Gemeinwohlziele und neue Organisationsformen untersucht. Hierfür werden unterschiedliche sozialwissenschaftliche Debattenstränge miteinander verknüpft und auf materiell-sachliche, räumliche, zeitliche und akteursbezogene Fragen der Energiewende bezogen. Damit erlaubt das Analysewerkzeug eine integrative Betrachtung von energiepolitischen Konflikten, die in dieser Kombination bisher noch nicht vorgenommen wurde.

Schlüsselwörter Energiewende · Konfliktanalyse · Gemeinwohl $\cdot$ Neue Organisationsformen

\section{Analysing Local Energy Conflicts: Outline of an Analysis Tool}

Abstract The success of the German energy transition is highly depending on developments at the local level. However, these local transitions towards a sustainable energy supply do not always run smoothly. Instead, their implementation is often troubled by local conflicts over renewable energy and new grid infrastructures. The question of public acceptance is linked to more fundamental questions such as the underlying objectives of their deployment as well as who benefits from these new facilities. These issues refer to more complex debates and negotiations on the public interest and new forms of organisations for power supply. This paper presents a tool for the analysis of local energy conflicts with special consideration of a notion of public interest and new forms of organisation. The tool links different strands of debate in the social sciences - regarding conflicts, modes of organisation and public interests - and relates them to material, spatial, temporal and actor-based 
issues of the energy transition. The analysis tool therefore allows an integrative reflection of energy conflicts, which has never been used in his combination so far.

Keywords Energy transition - Conflict analysis · Public interest $\cdot$ New forms of organisation

\section{Einleitung}

Die deutsche Energiewende besteht nicht nur aus einem Ausbau der dezentralen Strom- und Wärmeversorgung auf der Grundlage erneuerbarer Energieträger, sondern auch aus einer maßgeblichen Veränderung der Akteurslandschaft im Energiesektor. Zahlreiche neue Energiegenossenschaften, Neugründungen von kommunalen Energieversorgern und der Wandel von Konsumenten zu „Prosumenten“ (Verbraucher, die gleichzeitig auch Produzenten sind) stellen traditionelle Marktstrukturen in Frage (Matthes 2011). Sowohl bei Erzeugungsanlagen und Verteilnetzen als auch bei Akteuren und Organisationen lässt sich ein Aufeinandertreffen von neuen dezentralen Formen und traditionell zentral organisierten Strukturen beobachten. Schauplätze dieser "Großen Transformation“ (WBGU 2011) sind die Städte und Gemeinden, in denen zum einen bundes- und landespolitische Zielvorgaben umgesetzt werden, zum anderen sind diese aber auch der Ort von Aushandlungen, kollektiven Willensbildungsprozessen und natürlich auch Konflikten. Die Umbrüche im Zuge der Energiewende verwandeln die lokale Ebene daher, zum dritten, in einen Ort, in dem sich die politischen Herausforderungen der Energiewende nicht nur beobachten, sondern auch gestalten lassen. Insoweit entscheidet sich maßgeblich, ,vor Ort", ob der Umbau zu einer nachhaltigen Energieversorgung gelingt.

Nach wie vor stehen in der medialen sowie politischen Öffentlichkeit die Konflikte, die die Energiewende auf lokaler Ebene mit sich bringt, im Vordergrund, und selten wird auf die Verknüpfung mit lokalen Handlungsmöglichkeiten eingegangen. Dies ist der Ansatzpunkt des von 2013 bis 2016 laufenden Forschungsvorhabens „Lösung von lokalen energiepolitischen Konflikten und Verwirklichung von Gemeinwohlzielen durch neue Organisationsformen im Energiebereich (EnerLOG)“. Das Projekt untersucht am Beispiel von Rekommunalisierungen, Energiegenossenschaften und Bioenergiedörfern die Entstehung neuer Organisationsformen in der Energieversorgung und die damit verknüpften neuen Governance-Formen sowie den Beitrag, den diese zur Lösung von energiepolitischen Konflikten leisten können. In diesem Bericht aus Forschung und Praxis soll das erste konzeptionelle Ergebnis vorgestellt werden: ein Analysewerkzeug, welches die systematische und vergleichende Analyse von lokalen energiepolitischen Konflikten mit besonderer Berücksichtigung von neuen
Organisationsformen und Gemeinwohlaspekten zulässt. Damit werden verschiedene, bislang weitgehend nebeneinander stehende Debattenstränge verknüpft und so eine erweiterte Betrachtung energiepolitischer Konflikte ermöglicht. Die Rolle von - durchaus unterschiedlichen Gemeinwohlzielen, die implizit in vielen energiepolitischen Kontroversen (mit) verhandelt werden, kann damit sichtbar gemacht werden. Darüber hinaus werden neue Organisationsformen in der Energieversorgung, die ein wichtiges Element der Energiewende darstellen, als Gegenstand, aber auch als Lösungsmöglichkeit für Konflikte diskutiert. Gleichzeitig bietet das Analysewerkzeug eine Grundlage, einen Vergleich verschiedener lokaler Energiekonflikte vorzunehmen und damit auch allgemeingültige Leitlinien im Sinne einer „Best Practice“ im Umgang mit Konflikten zu entwickeln.

Grundlage des Textes ist eine Auswertung der Literatur zu Konflikten, Gemeinwohl und neuen Organisationsformen in der Energieversorgung sowie einige erste empirische Beobachtungen im Land Brandenburg. Der Beitrag führt zunächst in die Rolle von neuen Organisationsformen und Gemeinwohlzielen in der Energiewende ein (Kap. 2), stellt anschließend ausgewählte Beispiele für lokale energiepolitische Konflikte und Ansätze der sozialwissenschaftlichen Konfliktanalyse vor (Kap. 3), um dann das Analysewerkzeug für energiepolitische Konflikte darzustellen (Kap. 4). Im Fazit (Kap. 5) diskutieren wir Möglichkeiten und Grenzen des vorgestellten Werkzeugs, ziehen Schlussfolgerungen für die Anwendung und Weiterentwicklung des Instruments und formulieren weiteren Forschungsbedarf.

\section{Neue Organisationsformen und Gemeinwohlziele in der Energiewende}

\subsection{Neue Organisationsformen}

Die Energiewende umfasst nicht nur das Errichten von Windrädern, die Installation von Solaranlagen oder die Nutzung von Biomasse für die Erzeugung von Elektrizität und Wärme. Vielmehr umfasst sie auch neue Akteure wie Ökostromanbieter, neue lokale Kooperationsformen wie Bioenergiedörfer und „neue“ Unternehmens- und Eigentumsformen wie Stadtwerke und Energiegenossenschaften. Die damit umrissene Entstehung neuer Akteure in der Energieversorgung fassen wir unter dem Begriff der „,neuen Organisationsformen“ zusammen. Damit sind neue Rechts-, Betriebs- und Eigentumsformen im Energiesektor gemeint. Die neuen Organisationsformen in diesem Sinne müssen keine gänzlich neuen Organisationen sein; dies gilt umso mehr, als z. B. Stadtwerke, Energiegenossenschaften, aber auch technisch oder betrieblich autarkie- 
orientierte „Insellösungen“ zum Teil eine über 100-jährige Geschichte haben, die bis in die Frühzeit der Elektrisierung zurückreicht. So wurden zu Beginn des Stromzeitalters häufig in jenen Gebieten dezentrale Lösungen entwickelt, in denen die damals entstehenden größeren Energiekonzerne nicht aktiv waren. Während Letztere sich zunächst auf die Ballungsräume konzentrierten, entstanden in schwer zugänglichen Bergregionen oder in peripheren Kleinstädten Genossenschaften und lokale Werke zur Selbstversorgung mit Energie, die teilweise noch heute bestehen (Hausman/ Hertner/Wilkins 2008).

Die Energiewende verknüpft eine technologisch getriebene Dynamik der Dezentralisierung mit kleineren, neuartigen Unternehmen, die von Bürgern, der öffentlichen Hand oder von privaten Kleininvestoren getragen werden. Allein die Quantität dieser Neugründungen rechtfertigt es, hier von ,neuen“ Organisationsformen zu sprechen. Energiegenossenschaften erlebten seit der Einführung des Erneuerbare-Energien-Gesetzes (EEG) im Jahr 2000 einen Boom. In den Jahren 2006 bis 2014 wurden knapp 900 Energiegenossenschaften neu registriert (Müller/Holstenkamp 2015, S. 8). Im Jahr 2012 lag die aggregierte Mitgliederzahl aller deutschen Energiegenossenschaften bei 136.000, die durchschnittliche Einlage pro Mitglied betrug 3.165 Euro (DRGV 2013, S. 9). Dieser auch ökonomisch beachtliche Trend zu neuen gemeinschaftlichen Organisationsformen wird von zahlreichen Rekommunalisierungen im Energiesektor begleitet (Bauer 2012; Reck 2012; vgl. allgemein auch Cumbers 2012). Allein in den Jahren 2005 bis 2012 wurden insgesamt 72 in der Energieversorgung aktive Stadtwerke neu gegründet (Berlo/Wagner 2013). Dabei sind andere Rekommunalisierungsformen nicht erfasst, wie z. B. die Erweiterung des Aufgabenbereichs von Stadtwerken auf die Strom- oder Wärmeversorgung oder der Rückkauf von Anteilen an existierenden Netzbetreibern (Becker/Gailing/ Naumann 2012). Die Entstehung dieser neuen Organisationsformen stellt damit eine Ausdifferenzierung und teilweise auch eine Abschwächung des Privatisierungstrends der 1990er-Jahre dar, wie er sich in Folge der Liberalisierung der Strommärkte (Bontrup/Marquardt 2010) eingestellt hat.

Doch wie wird die Entstehung neuer Organisationsformen in der Forschung diskutiert? In vielen Publikationen werden Energiegenossenschaften als wichtiger Bestandteil einer Energiewende unter Einbeziehung der Bürger dargestellt (Yildiz/Rommel/Debor et al. 2015) und Rekommunalisierungen gelten als Möglichkeit, zunächst den kommunalen Einfluss auf Energieinfrastrukturen zu erhöhen (Bauer 2012). Dass die Entstehung bzw. die (gegebenenfalls veränderten) Tätigkeiten neuer Stadtwerke und Energiegenossenschaften schon für sich genommen zu Konflikten führen können, wird dagegen wenig beleuchtet. Die Entstehung neuer Organisationsformen wie auch die Per- sistenz bestehender Versorgungsstrukturen sind einerseits selbst Gegenstand von Konflikten: Rekommunalisierungen sind kommunalpolitisch mitunter heftig umstritten, Energiegenossenschaften oder Bioenergiedörfer können eine Reaktion auf die Unzufriedenheit mit bisherigen Grundversorgern sein und überzeugen nicht immer alle Einwohner. Andererseits können neue Organisationsformen dazu beitragen, verschiedene politische Ansprüche an die Energieversorgung umzusetzen, wie z. B. die Erreichung von Klimaschutzzielen und die Steigerung von demokratischem Einfluss und Kontrolle. Solche Ziele operationalisieren wir hier als Gemeinwohlaspekte der Energiewende.

\subsection{Gemeinwohlaspekte der Energiewende}

Als eindeutig positiv besetzter Begriff ist das Gemeinwohl gleichwohl politisch geladen und umstritten. Verschiedene Akteure nehmen für sich in Anspruch, im Sinne des Gemeinwohls zu handeln. Entgegen der alltagssprachlichen Verwendung, welche das Gemeinwohl als etwas versteht, was „allen“ zugutekommt und deswegen klar bestimmt ist, gehen neuere Forschungen davon aus, dass erst in gesellschaftlichen Aushandlungsprozessen festgelegt wird, was als „im Sinne des Gemeinwohls“ (Moss/Gudermann/ Röhring 2009) gelten kann. Diese Sichtweise bricht mit der Idee, dass Gemeinwohlziele universell bestehen und bereits vorgegeben sind. Gemeinwohl wird ,prozedural“ festgelegt (Moss/Gailing/Kern et al. 2013, S. 24). Dies kann in lokalen oder regionalen Gemeinschaften, in Leitbildprozessen oder im Stadtrat geschehen, aber auch im öffentlichen Raum oder ohne eine sichtbare „Arena“. Schließlich können gleichzeitig mehrere Vorstellungen davon existieren, was als Gemeinwohl zu betrachten ist. Der Bezug auf Gemeinwohlaspekte kann ebenso dazu dienen, partikulare Interessen zu verschleiern und durchzusetzen. Inwiefern das gelingt, hängt sowohl von den Ressourcen als auch der wahrgenommenen Legitimität der jeweiligen Akteure ab. Die Aushandlung des Gemeinwohls ist damit jedoch nicht zwingend ein gleichberechtigter Prozess, sondern ,zuallererst eine politische Frage, ihre jeweils konkrete Beantwortung Ausdruck gesellschaftlicher Kräfteverhältnisse" (Lederer/Naumann 2010, S. 109). Welche Vorstellungen vom Gemeinwohl existieren denn dann in Bezug auf die Energiewende?

Gemeinwohlziele spielen in den Debatten um die Umsetzung der Energiewende eine zentrale Rolle. Bei der Aushandlung der Transformation des Energiesystems geht es nicht nur um die Gewährleistung einer zuverlässigen, umweltschonenden und möglichst preiswerten Stromund Wärmeversorgung. Darüber hinaus werden mit der Energieversorgung politische Ziele des Klimaschutzes, der Sicherung von Arbeitsplätzen und allgemein der internationalen Wettbewerbsfähigkeit verknüpft. Vor diesem 
Hintergrund bietet die Diskussion von Gemeinwohlzielen einen normativen und analytischen Zugang zur Betrachtung der Energiewende.

Gemeinwohlziele sind dabei auf unterschiedlichen rechtlichen und räumlichen Ebenen verankert. Das deutsche Energiewirtschaftsgesetz (EnWG) nennt als Zweck der politischen Regulierung eine „möglichst sichere, preisgünstige, verbraucherfreundliche, effiziente und umweltverträgliche leitungsgebundene Versorgung der Allgemeinheit mit Gas und Elektrizität, die zunehmend auf erneuerbaren Energien beruht" (EnWG 2005, § 1) und eröffnet damit einen Katalog möglicher Gemeinwohlziele. Gleichzeitig stellt das Gesetz diesen Zielvorstellungen privatwirtschaftliche und marktbezogene Logiken (Ströbele/ Pfaffenberger/Heuterkes 2012) zur Seite, indem es im gleichen Paragraphen die „Sicherstellung eines wirksamen und unverfälschten Wettbewerbs bei der Versorgung mit Elektrizität und Gas“ (EnWG 2005, § 1) als Ziel postuliert. Auf lokaler Ebene können noch Anforderungen der Kommunalpolitik wie etwa regionale Wertschöpfung, der Erhalt oder die Schaffung von Arbeitsplätzen oder eine unabhängige Energieversorgung hinzukommen. Tabelle 1 stellt verschiedene gesellschaftliche Funktionen der Energieversorgung dar und ordnet diesen Gemeinwohlziele zu, die in Bezug auf die Energieversorgung artikuliert werden.

Die räumliche Abgrenzung der Geltung von Gemeinwohl ist eine Frage, die auf lokaler Ebene besonders relevant ist. Wer ist die Gemeinschaft, auf die sich Gemeinwohlüberlegungen beziehen oder die Gemeinwohlziele konkret aushandelt? Bezieht der Wunsch nach Teilhabe nur die Dorfgemeinschaft ein oder ebenso die angrenzende Kleinstadt? So lassen sich unterschiedliche räumliche Bezüge bzw. Geltungsbereiche des Gemeinwohls in der Energieversorgung identifizieren, die zu vielfältigen Konflikten führen können:

1. Maßstabsebenen der Betrachtung: Während der Ausbau erneuerbarer Energieträger auf nationaler und internationaler Ebene für den Klimaschutz und damit für nachhaltige Entwicklung insgesamt von zentraler Bedeutung ist und deshalb kaum in Frage gestellt wird, können auf lokaler und regionaler Ebene durchaus negative Effekte für Flora und Fauna auftreten.

2. Dimensionen von Nutzen bzw. Schäden: Die Errichtung von Windenergieanlagen beispielsweise kann einerseits dazu beitragen, das Steueraufkommen in Kommunen zu verbessern, andererseits befürchten Anwohner die Minderung des Wertes von Haus- und Grundeigentum. Darüber hinaus kann der materielle Nutzen von Energieerzeugungsanlagen ideellen Werten wie gewachsenen Kulturlandschaften entgegenstehen.

3. Leit- und Landschaftsbilder: Der Beitrag der Energieversorgung für das Gemeinwohl wird ebenfalls durch grundlegende Vorstellungen über die Wahrnehmung
Tab. 1 Gesellschaftliche Funktionen und Gemeinwohlziele in der Energieversorgung. (Quelle: Eigene Darstellung nach Naumann/Moss 2012)

Gesellschaftliche Funktion Artikulierte Gemeinwohlziele

Strukturpolitische Gewährleistung einer zuverlässigen

Funktionen (u. a. auch im Versorgung mit Elektrizität, Wärme engeren Sinne energieund Gas

politische Funktionen)

Steigerung der Unabhängigkeit von Energie- und Rohstoffimporten Versorgungsunternehmen als regionaler Investor, Arbeitgeber und Träger von Innovationen

Umweltpolitische Funktionen

Verminderung von Treibhausgasemissionen

Schonung von fossilen Ressourcen Ermöglichung einer allmählichen Abkehr von den Risiken atomarer Stromproduktion

Sozialpolitische

Funktionen

Gesellschaftliche Teilhabe

Vermeidung gesundheitlicher Risiken durch die Strom- und Wärmeerzeugung Sozialverträgliche Energiepreise

Demokratiepolitische

Funktionen Förderung von kommunaler Selbstverwaltung, demokratischer Teilhabe und bürgerschaftlichem Engagement

Haushaltspolitische

Funktionen

Symbolische Funktionen

Entlastung kommunaler Haushalte

Stärkung der Identitäts- und Profilbildung von Städten und Regionen durch lokal und regional verankerte Ver- und Entsorgungsunternehmen

und Entwicklung einer Region bzw. einer Landschaft bestimmt. Tourismus- oder Gesundheitsregionen stellen andere Anforderungen an die Planung von Energieanlagen als stärker agrarisch oder industriell geprägte Regionen.

In der Gestaltung lokaler Energiewenden spielen damit Gemeinwohlziele, deren Aushandlung bzw. deren Konkretisierung und die Formen ihrer Umsetzung eine wichtige Rolle. Die Gegenüberstellung verschiedener Gemeinwohlziele auf verschiedenen räumlichen Ebenen steht beispielhaft für die konfligierenden ökonomischen, ökologischen, sozialen und politischen Ansprüche, die mit der Energiewende verbunden sind. Darüber hinaus kann auch die Frage heftig umstritten sein, wie es gelingen kann, mehrere dieser Ziele gleichzeitig zu erreichen. Während verschiedene Gemeinwohlziele in öffentlichen Diskussionen nebeneinander stehen, ist ihre Differenzierung ein erster Schritt, um Widersprüche sichtbar und diejenigen Einzelinteressen nachvollziehbar zu machen, die sich hinter manchem Gemeinwohlargument verstecken. Die Bestimmung von Gemeinwohlaspekten und deren Abgrenzung von Partikularinteressen wird damit zu einer methodisch relevanten Herausforderung für die raumbezogene Sozialforschung. 
Neue Organisationsformen in der Energieversorgung treten vielfach mit dem Anspruch an, Gemeinwohlziele glaubwürdiger als die bisherigen, häufig privaten Unternehmen zu vertreten. Lokale energiepolitische Konflikte können daher letztendlich auch als Konflikte verstanden werden, in denen es gleichzeitig um neue Organisationsformen und die Erreichung bzw. Verwirklichung von Gemeinwohlzielen geht. Es ist daher wichtig, diese drei Felder (lokale Konflikte, neue Formen der Organisation und Verwirklichung von Gemeinwohlansprüchen) zusammenhängend zu betrachten. Damit wenden wir uns nunmehr einigen Merkmalen dieser Konflikte zu.

\section{Merkmale lokaler energiepolitischer Konflikte}

\subsection{Drei Beispiele aus Brandenburg}

Lokale Energiekonflikte entzünden sich an Veränderungen, häufig der Installation von neuen Erzeugungsanlagen oder der Realisierung einer neuen Stromtrasse, adressieren dann jedoch darüber hinausgehende Fragen nach politischer und wirtschaftlicher Teilhabe. Auch wenn in diesen Konflikten die Notwendigkeit der Energiewende grundsätzlich nur selten in Frage gestellt wird, so ist deren lokale Umsetzung dennoch zum Teil heftig umstritten. Kontrovers sind die Priorisierung verschiedener Gemeinwohlziele wie auch deren konkrete Verankerung in der Energieversorgung. Welche Form der Energieversorgung zum „Wohle aller“ ist, steht ebenso wenig fest, wie die Organisationsform der Energieversorgung. Das illustrieren die drei folgenden Beispiele aus Brandenburg.

a. „Hände weg vom Liepnitzwald!“ - Konflikte um Windräder

Im nördlich von Berlin gelegenen Liepnitzwald (Landkreis Barnim) stößt die Ausweisung von Flächen als Windeignungsgebiet auf erheblichen Protest. Von der Bürgerinitiative „Hände weg vom Liepnitzwald!“ wird nicht nur die geplante Ansiedlung von Windrädern in einem Erholungs- und Landschaftsschutzgebiet kritisiert, sondern auch die mit der Ausweisung von Windeignungsgebieten einhergehende Beschränkung der kommunalen Gestaltungsmöglichkeiten durch den regionalen Planungsträger. Darüber hinaus wird gefordert, dass die Windenergieanlagen nur genehmigt werden, wenn die Betreibergesellschaft sich auch am jeweiligen Standort ansiedelt. Zudem werden negative Auswirkungen wie etwa Lichtschattenschlag, Beeinträchtigung des Landschaftsbildes, Lärmbelästigungen, aber auch die Wertminderung von Grundstücken und Gebäuden befürchtet und als Argumente gegen die Errichtung der Windräder angeführt (Bürgerinitiative „Hände weg vom Liepnitzwald“ 2015). b. „Hochspannung tief legen“ - Konflikte um die Errichtung von Stromleitungen

Die Bürgerinitiative „Hochspannung tief legen“ im Landkreis Prignitz wendete sich, am Ende mit Erfolg, gegen die geplante Errichtung einer neuen Hochspannungs-Freileitung. Unter dem Motto „Für eine Prignitz mit Weitblick“ setzte sich die Initiative für die Verlegung von Erdkabeln ein. Die grundlegende Ablehnung von Freileitungen wurde mit befürchteten Wertminderungen für Haus- und Grundeigentümer, Belangen des Naturschutzes und möglichen gesundheitlichen Gefährdungen der Bevölkerung begründet. Trotz zahlreicher Einwendungen von Anwohnern und Kommunen wurde an der geplanten Freileitung zunächst lange Zeit mit dem Verweis auf die zusätzlichen Kosten einer anderen Lösung - festgehalten. Mittlerweile hat der regionale Netzbetreiber E.ON edis jedoch von der geplanten Freileitung Abstand genommen und plant stattdessen eine Erdkabellösung (Bürgerinitiative „Hochspannung tief legen“ 2015).

\section{c. Das Schlalacher Modell - Neue Beteiligungsformen zur Lösung von Konflikten}

In der Gemeinde Schlalach im Landkreis PotsdamMittelmark wurde hingegen gezeigt, wie man mit einer lokalen Lösung einen Konflikt produktiv gestalten kann. Nach der Ausweisung eines Windeignungsgebiets durch die Regionale Planungsstelle Havelland-Fläming schlossen sich hier die Grundstückseigentümer zu einer Arbeitsgruppe „Windkraft in Schlalach“ zusammen. Gleich zu Beginn wurde eine Bürgerversammlung einberufen, im Verlauf des Prozesses kristallisierte sich jedoch eine bislang ungewöhnliche Lösung heraus. Die Anwohner der Gemeinde entschieden sich, den Bauherrn und den Betreiber der Fläche selbst auszuwählen und durch ein Flächenpachtmodell auch diejenigen Grundeigentümer an den Einnahmen zu beteiligen, auf deren Flächen keine Produktionsanlagen entstehen. Zusätzlich wird ein Teil der Gewinne einer Stiftung zur Verfügung gestellt, die in der Sicherung der lokalen Daseinsvorsorge tätig ist. So wird die Grundstruktur kleiner Parzellen in ein Modell verwandelt, das der Allgemeinheit im Ort zugutekommt (MWE 2011).

Diese Beispiele zeigen: Lokale Konflikte verschiedener Art und Ausprägung sind Teil der Energiewende. Sie kristallisieren sich entlang unterschiedlicher Veränderungen, die mit der Energiewende zusammenhängen. Der Aufbau neuer Anlagen geht mit der Frage einher, wer über deren Errichtung bestimmen oder davon profitieren kann. Damit ist die Frage nach der Organisation, also nach der Steuerung und dem Eigentum an diesen Anlagen (mitunter nicht nur) impliziter Gegenstand dieser Konflikte. Das Beispiel Schlalach verdeutlicht hier eine andere Form des Interessenausgleichs, indem Organisationen geschaffen 
werden, die eine breitere Beteiligung an Erträgen aus neuen Windanlagen erlauben. Diese Art der Lokalisierung und der Verräumlichung eines Ausgleichsmechanismus spricht zugleich auch die räumliche Verteilung der Gewinne an. Nicht nur wer profitiert, sondern auch wo profitiert wird, ist eine Frage, die in lokalen Energiekonflikten aufbricht.

Die verschiedenen Argumente, die von den Kritikern dieser Anlagen hervorgebracht werden, beziehen sich zudem auf eine Reihe von Gemeinwohlzielen, die nicht alle aus im engeren Sinne energiepolitischen Fragen abgeleitet werden können. Umweltschutz, der „Erhalt der Heimat" oder der heimischen Kulturlandschaft, der Schutz von Vogelarten wie dem mittlerweile sprichwörtlichen Rotmilan verweisen ebenso auf andere schützenswerte Interessen, die über Einzelinteressen hinausgehen. Energiepolitische Konflikte sind damit häufig auch mit konkurrierenden Gemeinwohlvorstellungen (z. B. Klimaschutz vs. Schutz von bestehenden Landschaften) und Fragen von Organisation verknüpft, die sich jeweils auch räumlich artikulieren. Ziel unseres Beitrags ist es, diese verschiedenen Facetten und Dimensionen von lokalen Energiekonflikten mit einem integrativen Ansatz zu erfassen. Dabei greifen wir auf bestehende Forschungen zur sozialwissenschaftlichen Konfliktforschung zurück und erweitern diese um räumliche und energiespezifische Aspekte.

\subsection{Sozialwissenschaftliche Konfliktanalysen}

Seitdem in vielen Ländern der Ausbau und die Nutzung erneuerbarer Energieträger auf der politischen Agenda stehen, ist auch die sozialwissenschaftliche Literatur $\mathrm{zu}$ energiepolitischen Konflikten angewachsen (Marg/ Hermann/Hambauer et al. 2013; Otto/Leibenath 2013; Weiss 2013). Häufig auf konkreten Fallstudien aufbauend, hat sich eine breite, auch internationale Literatur zu lokalen Energiekonflikten entwickelt. Hierbei ist zu beobachten, dass sowohl neue Organisationsformen der Energieversorgung wie auch Gemeinwohlziele im Zusammenhang mit energiepolitischen Konflikten bisher nur am Rande behandelt werden. Zwei wichtige Stränge der sozialwissenschaftlichen Forschung zu energiepolitischen Konflikten sollen in diesem Zusammenhang genannt werden. Zum einen dreht sich ein großer Teil der akademischen Debatte um die Frage, inwiefern es gerechtfertigt ist, lokale Konflikte in erster Linie nach der Logik des NIMBYAnsatzes (benannt nach der Maxime „Not In My Back Yard“, der englischsprachigen Fassung des St.-FloriansPrinzips) zu beschreiben (z. B. Wolsink 2000; Van der Horst 2007; Jones/Eiser 2010). Der NIMBY-Ansatz geht davon aus, dass lokale Anwohner geplanter erneuerbarer Energieanlagen diese zwar prinzipiell befürworten, jedoch in ihrer unmittelbaren Umgebung ablehnen. Das muss zumeist als Vereinfachung gelten. Ein weiterer relevanter Strang der
Literatur in diesem Zusammenhang beschäftigt sich mit der Frage, inwieweit sich die wahrgenommene Gerechtigkeit auf die soziale Akzeptanz von erneuerbaren Energien auswirkt (Gross 2007; McLaren Loring 2007; Wolsink 2007; Ottinger/Hargrave/Hopson 2014). Hierfür spielt sowohl die Möglichkeit eine Rolle, den Entscheidungsprozess beeinflussen zu können, als auch die Chance, an den (späteren) Vorteilen der Anlagen partizipieren zu können.

In seinem Versuch, verschiedene Ansätze der sozialwissenschaftlichen Konfliktforschung zu kategorisieren, benennt Saretzki (2010) drei Dimensionen: a) Konfliktgegenstand, b) Konfliktparteien, c) Austragungsformen und Konfliktregelungen. Aus diesen beobachteten Herangehensweisen der sozialwissenschaftlichen Konfliktanalyse leitet Saretzki drei verschiedene Zugänge zu sozialen Konflikten ab:

1. Der gegenstandsbezogene Zugang nimmt sich der Frage an, worum es in dem Konflikt geht (,die Sache"). Neben der Klärung des Konfliktgegenstandes werden hier auch grundlegende Streitpunkte wie unterschiedliche Wertvorstellungen oder politische Überzeugungen typisiert. Zum anderen werden verschiedene Konflikttypen identifiziert wie beispielsweise Risiko-, System- oder Wertekonflikte. Auch bestimmt der gegenstandsbezogene Zugang, auf welcher politischen Ebene sich der Konflikt abspielt. So kann ein Technikkonflikt auf ein konkretes Technisierungsprojekt, auf einen bestimmten Technisierungspfad oder auf die Technikentwicklung insgesamt Bezug nehmen (Saretzki 2010, S. 39 f.).

2. Der akteursbezogene oder soziale Zugang analysiert Akteure, Akteurskonstellationen, Akteursstrategien und ihre Aktionen, Interaktionsformen und Konfliktaustragungsebenen. Dieser Zugang fragt, für wen ein bestimmter Sachverhalt zum Problem wird und wie sich andere Akteure dazu auf welche Weise äußern (Saretzki 2010, S. 42).

3. Der regelungsorientierte Zugang richtet den Blick in erster Linie auf mögliche Konfliktlösungen, das heißt auf Verfahren der Konfliktbearbeitung, -vermittlung und -regelung. Dieser Zugang ist deshalb zugleich von einem normativen Erkenntnisinteresse geleitet, da er „unerwünschte“ Formen der Konfliktaustragung statuiert, die sich auf eine - mitunter auch gewaltsame Unterdrückung des Konflikts oder derjenigen Akteure, die ihn ansprechen, beziehen (Saretzki 2010, S. 43).

Saretzkis Ansatz umfasst den Gegenstand, die Akteure sowie die Formen und Möglichkeiten, einen Konflikt auszutragen und ihn zu regeln. Saretzki liefert damit eine Kategorisierung für eine Vielzahl bestehender Zugänge in sozialwissenschaftlichen Konfliktanalysen, die generalisiert als Grundlage für eine allgemeine Konfliktanalyse dienen kann. Um die vielfältigen Aspekte und Besonderheiten lokaler energiepolitischer Konflikte zu erfassen, soll dieser 
allgemeine Zugang nun für die Analyse lokaler energiepolitischer Konflikte weiterentwickelt werden.

\section{Lokale energiepolitische Konflikte analysieren}

\subsection{Elemente der Analyse}

Der zentrale Beitrag dieses Artikels ist die Entwicklung eines Analysewerkszeugs, das zwei Ziele verfolgt. Einerseits soll die Entwicklung von lokalen energiepolitischen Konflikten, Gemeinwohlzielen und neuen Organisationsformen der Energieversorgung erfasst, andererseits deren komplexes Zusammenwirken betrachtet und verdeutlicht werden. Dafür entwickeln wir Saretzkis gegenstands- und akteursbezogenen Ansatz zu den Zugängen der sozialwissenschaftlichen Konfliktanalyse für unsere Zwecke weiter. Während der akteurszentrierte Zugang Saretzkis als Akteursdimension im Prinzip unverändert in unser Modell übernommen wird, sind für die Erfassung der gegenstandsbezogenen Seite von Konflikten drei weitere Schritte der Anpassung nötig: Erstens muss Saretzkis Konzeption auf den spezifischen Gegenstand von lokalen energiepolitischen Konflikten zugeschnitten werden. Dabei kommt, wie die oben angeführten Beispiele zeigen, materiellen, das heißt physischen Veränderungen bestehender Infrastrukturen eine große Relevanz zu. Zweitens haben wir in den vorangegangenen Kapiteln gezeigt, dass sowohl neue Organisationsformen wie auch die umsetzungsreife Konkretisierung von Gemeinwohlaspekten von Aushandlungsprozessen abhängen, die an einen gewissen Ort und eine gewisse Zeit gebunden sind. Deswegen schlagen wir eine stärker raumbezogene Betrachtung vor, als dies bei Saretzki der Fall ist. Drittens ist es uns wichtig, die wechselvolle Dynamik von Konflikten und Aushandlungsprozessen genauer zu erfassen, weswegen wir Saretzkis Ansatz um eine zeitliche Dimension erweitern. Der regelungsorientierte Zugang Saretzkis wird im nachfolgend entwickelten Analysewerkzeug hingegen nicht weiter verfolgt, da dieser den Fokus auf mögliche Verfahren der Konfliktbearbeitung, -vermittlung und -regelung legt, und dies nicht im Fokus des Analysewerkzeuges liegt. Damit entstehen vier Dimensionen, die sich sowohl für die Analyse von Konflikten selbst als auch für die Ermittlung der Bedeutung von Gemeinwohlzielen und neuen Organisationsformen verwenden lassen, wie sie innerhalb dieser Konflikte artikuliert werden. Diese verschiedenen Konfliktdimensionen werden im Folgenden vorgestellt und durch Beispiele aus der Literatur veranschaulicht.

\subsubsection{Die materielle Dimension}

Die materielle Dimension knüpft an Saretzkis gegenstandsbezogene Dimension an, ist jedoch nicht deckungsgleich mit dieser. Sie identifiziert den konkreten Gegenstand eines Konfliktes oder den Gegenstand, der den Auslöser des Konfliktes bildet, z. B. eine Windkraftanlage, ein auszubauendes Stromnetz oder eine geplante Biogasanlage. Die Berücksichtigung der materiellen Dimension in der Konfliktanalyse geht davon aus, dass es sich bei technischer Infrastruktur nicht nur um physisch-materielle Gegebenheiten handelt, sondern dass ihre Struktur und die mit ihr gegebenen Charakteristika die gesellschaftlichen Aushandlungsprozesse darum mit beeinflussen, und geht damit über Saretzkis gegenstandsbezogenen Zugang hinaus. Gleichzeitig kann das Ergebnis von Aushandlungsprozessen die materielle Veränderung von Infrastrukturen zum Ergebnis haben. Letztlich beziehen sich auch Überlegungen zu Gemeinwohlzielen und neuen Organisationsformen immer auf die konkreten Gegenstände zurück, die „geregelt" werden sollen. Ein Beispiel aus der Literatur zu dieser Art von Energiewendekonflikten ist die Arbeit von Zografos und Martínez-Alier (2009), die Auseinandersetzungen um Windenergie analysieren und das Fazit ziehen, dass die umstrittene neue technische Infrastruktur eine Materialisierung bestehender Machtverhältnisse ist, die zu ungleicher Verteilung von Kosten und Nutzen führt und daher konflikthaft wird.

\subsubsection{Die räumliche Dimension}

Die materielle Dimension ist eng verknüpft mit der räumlichen Dimension. Diese stellt Fragen danach ins Zentrum, ob sich der Konflikt um einen bestimmten Standort dreht, wie sich der Konflikt räumlich ausbreitet und ob er sich auf weitere räumliche Maßstabsebenen auswirkt bzw. von diesen beeinflusst wird, aber auch wie räumliche Bedingungen allgemein auf den Konflikt wirken. Die räumliche Dimension berührt auch Fragen nach dem räumlichen Bezug der Energieversorgung (Standorte, Einzugsbereiche und Landschaftsveränderungen) und deren räumlicher Governance (etwa die Forderung nach einem räumlichen Ausgleich von Kosten oder Erträgen in der Energieversorgung zwischen verschiedenen Teilräumen; vgl. Klagge 2013). Gleichzeitig sind räumliche Identitäten und Leitbilder eng mit Gemeinwohlzielen verknüpft, materielle und institutionelle Raumstrukturen können sich wiederum auf neue Organisationsformen auswirken. Welche Rolle die räumliche Dimension in Energiewendekonflikten spielen kann, wird in einer Studie von Jones und Eiser (2010) betont. Hier wird gezeigt, dass die räumliche Distanz von geplanten Onshore- und Offshore-Windenergieanlagen ein 
zentraler Bestimmungsfaktor dafür ist, wie die geplanten Anlagen durch die Bevölkerung bewertet werden.

\subsubsection{Die zeitliche Dimension}

Die zeitliche Dimension von Energiekonflikten ist ebenso eine zentrale Kategorie und bezieht sich auf die Entwicklungsgeschichte und den Verlauf des Konflikts. So lässt sich nachvollziehen, wie sich die Auseinandersetzung im Zeitverlauf geändert hat. Entwicklungspfade und Wendepunkte des Konflikts können aufgezeigt und Meilensteine, die zu neuen Organisationsformen führen, können benannt werden. Wendepunkte können die Gründung einer neuen Organisation sein oder eine Verschiebung in der öffentlichen Diskussion durch die Betonung von Gemeinwohlzielen. In der zeitlichen Dimension zeigen sich ferner auch „Ungleichzeitigkeiten“ im Sinne einer Überlappung von bisher Bestehendem und bevorstehendem Neuem. In der Literatur zu Energiewendekonflikten wird die zeitliche Dimension häufig beschrieben. Zum Beispiel arbeiten Ohlhorst und Schön (2010) den zeitlichen Verlauf der Windenergienutzung und den damit verbundenen Konflikten in Deutschland seit den 1970er-Jahren heraus.

\subsubsection{Die Akteursdimension}

Im Zentrum vieler Arbeiten zu Konfliktanalysen steht die Akteursdimension, von Saretzki als ,, akteursbezogene“ oder „soziale“ Dimension bezeichnet (Saretzki 2010, S. 42). Durch Auseinandersetzungen zwischen sowie innerhalb von Akteursgruppen werden Konflikte erst wahrnehmbar und ihre jeweiligen Interessen, Ressourcen und Strategien steuern die Konfliktentstehung, den Konfliktverlauf und -ausgang maßgeblich. Der Streit um Gemeinwohlziele und neue Organisationsformen ist dabei ohne Akteure nicht denkbar, sie müssen von ihnen formuliert und mit Leben gefüllt werden. Jegen und Audet (2011) beispielsweise analysieren „Advocacy Coalitions“ und arbeiten in ihrer Studie über die Auseinandersetzungen um Windkraft heraus, dass die Kontroverse um Windkraftanlagen auf einer Ablehnung des gewählten Entwicklungs- und Implementierungsmodells beruhen kann, Zustimmung und Ablehnung damit nicht nur eine Frage des „ob“, sondern auch des „wie“ der Anlagenerrichtung sind.

Wie die oben angeführten Beispiele andeuten, sind diese vier Dimensionen teilweise bereits Gegenstand empirischer Arbeiten zu Konflikten im Zusammenhang mit der Energiewende, wobei viele akteurszentrierte Untersuchungen zugleich die zeitliche Dimension einschließen. Auf die räumliche oder materielle Dimension fokussierende Forschungen sind hingegen noch weniger häufig anzutreffen. Der Grundstein für eine systematische Untersuchung aller vier Dimensionen, wie sie bisher in der Literatur noch nicht anzutreffen ist, ist mit dem hier vorgestellten Analysewerkzeug gelegt.

\subsection{Skizze des Analysewerkzeuges}

Das Analysewerkzeug operationalisiert die oben genannten vier Konfliktdimensionen für die Analyse unterschiedlicher lokaler energiepolitischer Konflikte, wobei explizit die Bedeutung von Gemeinwohlzielen und die Entstehung neuer Organisationsformen berücksichtigt werden. Lokale energiepolitische Konflikte, Gemeinwohlziele und neue Organisationsformen bilden damit die Kategorien für die Analyse, die im hier vorgestellten Analysewerkzeug in einer Matrix systematisch miteinander verknüpft werden (vgl. Tab. 2):

1. Lokale energiepolitische Konflikte (linke Spalte): Hier werden die unterschiedlichen Dimensionen von Konflikten untersucht, um der Komplexität von lokalen Aushandlungsprozessen gerecht zu werden.

2. Die Bedeutung von Gemeinwohlzielen (mittlere Spalte): Gemeinwohlziele stehen nicht fest, sondern sind Ergebnis politischer Auseinandersetzungen, ihre unterschiedliche materielle, räumliche und zeitliche Ausprägung sowie die jeweilige Rolle von Akteuren werden so in den Blick genommen.

3. Die Rolle neuer Organisationsformen (rechte Spalte): Bei lokalen Konflikten und der Bestimmung von Gemeinwohlzielen sind neue Organisationsformen häufig Bestandteil. Worin diese Rolle genau besteht, wird im Rahmen dieser Kategorie analysiert.

Diese drei Kategorien werden auf die oben herausgearbeiteten vier Analysedimensionen bezogen. In dieser Verknüpfung der Kategorien mit den Dimensionen liegt der Mehrwert des Analysewerkzeuges: Die Kombination der unterschiedlichen konzeptionellen Ebenen verspricht interessante Schlussfolgerungen, die aus einer simplen Konfliktanalyse (etwa allein durch die Frage ,wer will was?") nicht abzuleiten sind. Das Analysewerkzeug erlaubt dabei eine Verknüpfung der Konflikt- und Gemeinwohlanalyse mit der Entstehung neuer Organisationsformen.

Um diese analytischen Verknüpfungen direkt auf empirische Fragestellungen anwendbar zu machen, wurden $\mathrm{zu}$ jeder Verknüpfung von Kategorie und Dimension (z. B. die materielle Dimension von Gemeinwohlzielen) forschungsleitende Fragestellungen entworfen. In der Praxis wird das Analysewerkzeug derart verwendet, dass alle in der Matrix enthaltenen Fragen im jeweiligen Konfliktfall abgefragt und Bezüge zwischen den verschiedenen Kategorien geprüft werden. Die möglichen Querverbindungen zwischen den verschiedenen Themenfeldern sollen vor schnellen Schlüssen warnen, wie zum Beispiel, dass durch die Gründung neuer Organisationsformen ein Konflikt auto- 
Tab. 2 Skizze eines Analysewerkzeugs. (Quelle: Eigene Darstellung) Lokale energiepolitische Konflikte Bedeutung von artikulierten Gemeinwohlzielen Rolle neuer Organisationsformen Materielle Um welchen konkreten Gegenstand Inwiefern werden im Konflikt Gemeinwohlziele Wie werden welche materiellen Dimension geht es im Konflikt? explizit thematisiert? Objekte organisiert und (z. B. rechtlich) reguliert?

Mit welchen anderen Gegenständen steht der Konflikt im Zusammenhang?

Räumliche Welche Rolle spielt der konkrete Dimension Standort im Konflikt?

Welche räumliche Ausbreitung hat der Konflikt und hat er sich im Konfliktverlauf verschoben?

Wie wirken räumliche Bedingungen auf den Konflikt?

Zeitliche Dimension

Welche zeitlichen Entwicklungsstufen und Wendepunkte des Konflikts gibt es? Inwieweit hat sich der Gegenstand des Konfliktes verändert?
Welche Anlagen, Netze und Ressourcen werden Welche Forderungen für deren Remit welchen Gemeinwohlzielen verbunden?

Organisation bestehen?

Auf welches Gebiet bzw. auf welche räumliche Einheit beziehen sich die Gemeinwohlziele?

Mit welchen räumlichen Identitäten, Entwicklungszielen oder Leitbildern sind die Gemeinwohlziele verbunden?

Welche räumliche Ausbreitung bzw. welche Einzugsbereiche haben (neue) Organisations-formen und ihre Teilhaber?

Wie ist die neue Organisation räumlich verflochten?

Wie wirken räumliche Bedingungen auf die neue Organisationsform, wie wirkt die neue Organisationsform auf diese Bedingungen?

Welche Gemeinwohlziele spielten in der lokalen Energieversorgung in der Vergangenheit eine Rolle? Wie wurden sie verwirklicht? Wie haben sich diese Ziele verändert?

Welche Organisationsform bestand zuvor?

Wann und womit wurde die Entstehung der neuen Form eingeleitet? Welche unterschiedlichen Vorstellungen gab es hinsichtlich von Organisationsformen?

Haben die Gemeinwohlziele einen historischen

Welche wichtigen Schritte (inkl. Bezug, oder sind sie auf Gegenwart oder Zukunft Rückschritte) und Wendepunkte gab gerichtet?

es im organisatorischen Wandel?

Welche Gemeinwohlziele werden von welchen Welche Akteure entscheiden Akteuren artikuliert und wie erfolgt das?

Inwieweit werden Gemeinwohlziele in Anspruch genommen, um eigene Interessen und Standpunkte zu legitimieren? Konflikt direkt oder indirekt beteiligt?

Was sind ihre Interessen, Ressourcen und Strategien (z. B. Eingehen von Bündnissen)?

Welche Akteure können sich in welchen Konstellationen im Konflikt durchsetzen?

bei der Entstehung der neuen Organisationsform?

Welche Akteure werden ausgeschlossen?

Welche Akteure können ihre Ressourcen- oder ihre strategische Position verbessern? Welche Beweggründe wirken für bzw. gegen die neue Organisationsform? matisch gelöst wird oder dass ein Stadtwerk automatisch dem Gemeinwohl verpflichtet ist. Beispiele für solche Querverbindungen sind etwa, dass neue Organisationsformen sowohl Auslöser, Gegenstand als auch Ergebnis von Konflikten sein können. Gemeinwohlziele können in der Organisationsform verankert und mit ihr vereinbar sein, als auch ihr grundlegend widersprechen. Die Verfolgung von Gemeinwohlzielen kann bei der Lösung von Konflikten eine wichtige Rolle spielen, aber auch zu neuen bzw. weiteren Konflikten führen.

Das Analysewerkzeug kann auf unterschiedliche Typen energiepolitischer Konflikte bezogen werden und deren Bezug zu Gemeinwohlzielen sowie die Rolle, die neue Organisationsformen dabei spielen, untersuchen. Exemplarisch hierfür stehen die folgenden fünf Konflikt- typen, die auf unterschiedliche Weise Gemeinwohlziele und neue Organisationsformen berühren und somit verdeutlichen, wie das Analysewerkzeug auch dafür angewendet werden kann, energiepolitische Konflikte zu kategorisieren. Die angeführten Beispiele ließen sich erweitern und betreffen Gegenstände, die bei Konflikten besonders häufig thematisiert werden.

1) Verteilungskonflikte - und deren Beilegung - sind ein zentrales Thema bei der Aushandlung von Gemeinwohlzielen wie auch bei der Gründung neuer Organisationsformen. Ein häufiges Beispiel ist hierfür die Beteiligung an den Erträgen von Anlagen erneuerbarer Energien.

2) Verfahrenskonflikte berühren sowohl Gemeinwohlziele als auch neue Organisationsformen bei der Suche nach 
geeigneten partizipativen Verfahren, zum Beispiel bei der Festlegung von Anlagenstandorten, sowie beim Versuch einer dauerhaften Verankerung dieser Verfahren in Organisationen der Energieversorgung.

3) In Standort- und Landnutzungskonflikten treffen unterschiedliche Vorstellungen von Gemeinwohl aufeinander. An diesen Konflikten können die organisatorischen Träger der Energieversorgung ebenfalls beteiligt sein. Bei der Neugründung von Organisationen besteht aber auch die Hoffnung, dass neue Organisationsformen helfen können, einen Ausgleich zwischen unterschiedlichen Vorstellungen hinsichtlich der Errichtung von Anlagen und Netzinfrastrukturen zu finden.

4) Identitätskonflikte betreffen das Selbstverständnis von Orten und Regionen, aber auch von Energieversorgungsunternehmen. Städte und Dörfer können sich beispielsweise der Nutzung erneuerbarer Energien verpflichtet fühlen. Entsprechend dieses Selbstverständnisses werden unterschiedliche Gemeinwohlziele für die Energieversorgung definiert, die die Wahl sowie auch die Gestaltung der Organisationsform bestimmen können.

5) Energieträgerkonflikte bzw. technologische Konflikte können ebenfalls für jeweils unterschiedliche Gemeinwohlziele bzw. -vorstellungen stehen (z. B. Erhalt von Arbeitsplätzen in der bestehenden Energieversorgung vs. Erreichung von Klimaschutzzielen), die dann in verschiedene Organisationsformen eingeschrieben werden bzw. zu Konflikten zwischen und innerhalb von Organisationen führen können.

Diese Auswahl verdeutlicht, dass unterschiedliche Typen von energiepolitischen Konflikten vielfältig mit Gemeinwohlzielen und neuen Organisationsformen verknüpft sein können. Daher bietet das hier vorgestellte Analysewerkzeug einen Zugang zu Fragen des übergeordneten Gemeinwohls, deren organisatorischer Verankerung, zu zeitlichen und räumlichen Aspekten des Konflikts und ihrem Einfluss auf dessen Verlauf, und schließlich einen Zugang zu den Eigenschaften und Rollen der Akteure sowie des konkreten Konfliktgegenstands. Die explizite Verknüpfung dieser Aspekte kann dazu beitragen, die Besonderheiten lokaler Konflikte zu verstehen, Begründungszusammenhänge entlang von Gemeinwohlansprüchen nachzuvollziehen und den institutionellen Wandel der Energieversorgung zu erfassen.

\section{Fazit}

Das hier vorgestellte Analysewerkzeug kann dazu beitragen, energiepolitische Konflikte mit übergeordneten Fragen nach Gemeinwohl und neuen Organisationsformen zu verbinden. Damit wird der Vielschichtigkeit und mitunter
Widersprüchlichkeit von energiepolitischen Konflikten Rechnung getragen. Während das Analysewerkzeug einen neuen Ansatz bietet, sind jedoch auch die Grenzen seiner Anwendung $\mathrm{zu}$ benennen. Zum einen treten energiepolitische Konflikte nicht abgekoppelt, sondern überlagert von Auseinandersetzungen in anderen gesellschaftlichen Bereichen auf. Zum anderen können Konflikte auch latent verlaufen und Interessengegensätze nicht immer offen zu Tage treten. Für die Anwendung und Weiterentwicklung des Analysewerkzeuges ergeben sich daher verschiedene weitergehende, empirisch noch zu prüfende Fragen:

- Welche gemeinsamen bzw. voneinander abweichenden Muster lassen sich für lokale energiepolitische Konflikte z. B. in ländlichen bzw. städtischen Räumen feststellen?

- Haben verschiedene Typen neuer Organisationsformen einen Einfluss auf den Verlauf und die Ergebnisse von lokalen energiepolitischen Konflikten? Welche Rolle spielen unterschiedliche Gemeinwohlverständnisse?

- Gibt es Akteurskonstellationen oder räumliche Kontextfaktoren, die das Entstehen einer bestimmten Organisationsform begünstigen?

- Worin liegen empirische oder systematische Grenzen von Bürgerbeteiligung, die häufig als Mittel zur Lösung von Konflikten angesehen wird?

- Wie lässt sich das Analysewerkzeug auf Beispiele aus dem Ausland anwenden, die von einem ganz anderen Kontext als den hier zugrunde liegenden Rahmenbedingungen der bundesdeutschen Energiewende geprägt sind?

Die Anwendung des Analysewerkzeugs und die Diskussion dieser Fragen können somit einen Beitrag für die Ermittlung der örtlichen Verteilung von Lasten und Nutzen und somit für die Ermittlung von Gewinnern und Verlierern lokaler Energiewenden leisten. Gemeinwohl kann hierbei ebenso eine Rahmung für die Betrachtung wie auch eine Orientierung für die Lösung von Konflikten darstellen. Darüber hinaus können empirische Untersuchungen lokaler energiepolitischer Konflikte konzeptionelle Debatten der sozialwissenschaftlichen Konfliktforschung, der Institutionen-, Gemeinwohl- und Gemeinschaftsgüterforschung wie auch der Energieforschung insgesamt anregen.

\section{Literatur}

Bauer, H. (2012): Zukunftsthema „Rekommunalisierung“. In: Die Öffentliche Verwaltung 65 (9), 329-338.

Becker, S.; Bues, A.; Naumann, M. (2014): Die Analyse lokaler energiepolitischer Konflikte und das Entstehen neuer Organisationsformen. Theoretische Zugänge und aktuelle Herausforderungen. Erkner/Freiburg/Potsdam. $=$ EnerLOG Working Paper. 
Becker, S.; Gailing, L.; Naumann, M. (2012): Neue Energielandschaften - neue Akteurslandschaften. Eine Bestandsaufnahme im Land Brandenburg. Berlin. = rls Studien.

Berlo, K.; Wagner, O. (2013): Stadtwerke-Neugründungen und Rekommunalisierungen. Energieversorgung in kommunaler Verantwortung. Bewertung der 10 wichtigsten Ziele und deren Erreichbarkeit. Wuppertal.

Bontrup, H.-J.; Marquardt, R.-M. (2010): Kritisches Handbuch der deutschen Elektrizitätswirtschaft. Branchenentwicklung, Unternehmensstrategien, Arbeitsbeziehungen. Berlin. $=$ Forschung aus der Hans-Böckler-Stiftung, 112.

Bürgerinitiative „Hände weg vom Liepnitzwald!“ (2015): Die Bürgerinitiative stellt sich vor. http://www.pro-liepnitzwald.de/downloads/Darstellung_BI.pdf (02.03.2015).

Bürgerinitiative „Hochspannung tief legen“ (2015): Hintergrundinformationen. http://hochspannungtieflegen.wordpress.com/ category/hintergrundinformationen/ (02.03.2015).

Cumbers, A. (2012): Reclaiming Public Ownership. Making Space for Economic Democracy. London.

DRGV - Deutscher Raiffeisen- und Genossenschaftsverband (2013): Energiegenossenschaften. Ergebnisse der Umfrage des DRGV und seiner Mitgliedsverbände. http://www.dgrv.de/webde.nsf/7d5e59ec98 e72442c1256e5200432395/dd9db514b5bce595c1257bb200263bbb/ \$FILE/Umfrageergebnisse\%20Energiegenossenschaften.pdf (02.03.2015).

EnWG - Energiewirtschaftsgesetz (2005): Gesetz über die Elektrizitäts- und Gasversorgung vom 07.07.2005. http://www. gesetze-im-internet.de/bundesrecht/enwg_2005/gesamt.pdf (Zugriff am 02.03.2015).

Gross, C. (2007): Community perspectives of wind energy in Australia: The application of a justice and community fairness framework to increase social acceptance. In: Energy Policy 35 (5), 2683-2691.

Hausman, W. J.; Hertner, P.; Wilkins, M. (Hrsg.) (2008): Global Electrification. Multinational Enterprise and International Finance in the History of Light and Power, 1878-2007. Cambridge. = Cambridge Studies in the Emergence of Global Enterprise.

Jegen, M.; Audet, G. (2011): Advocacy coalitions and wind power development: Insights from Quebec. In: Energy Policy 39 (11), 7439-7447.

Jones, C. R.; Eiser, R. J. (2010): Understanding, local' opposition to wind development in the UK: How big is a backyard? In: Energy Policy 38 (6), 3106-3117.

Klagge, B. (2013): Governance-Prozesse für erneuerbare Energien: Akteure, Koordinations- und Steuerungsstrukturen. In: Klagge, B.; Arbach, C. (2013) (Hrsg.): Governance-Prozesse für erneuerbare Energien. Hannover, 7-17. = Arbeitsberichte der ARL, 5.

Lederer, K.; Naumann, M. (2010): Öffentlich, weil es besser ist? Politische Gemeinwohlbestimmung als Voraussetzung einer erfolgreichen Kommunalwirtschaft. In: Berliner Debatte Initial 21 (4), 105-116.

Marg, S.; Hermann, C.; Hambauer, V.; Becké, A. B. (2013): „Wenn man was für die Natur machen will, dann stellt man da keine Masten hin" - Bürgerproteste gegen Bauprojekte im Zuge der Energiewende. In: Marg, S.; Geiges, L.; Butzlaff, F.; Walter, F. (Hrsg): Die neue Macht der Bürger. Was motiviert die Protestbewegungen? BP-Gesellschaftsstudie. Reinbek, 94-138.

Matthes, F. C. (2011): Strommärkte als Auslaufmodell? Die Rolle und das Design von Marktmechanismen in der „Großen Transformation" des Stromversorgungssystems. In: Schuetz, D.; Klusmann, B. (Hrsg.): Die Zukunft des Strommarktes. Anregungen für den Weg zu $100 \%$ erneuerbare Energien. Bochum, 85-106.

McLaren Loring, M. J. (2007): Wind energy planning in England, Wales and Denmark: Factors influencing project success. In: Energy Policy 35 (4), 2648-2660.
Moss, T.; Gailing, L.; Kern, K.; Naumann, M.; Röhring, A. (2013): Energie als Gemeinschaftsgut? Anregungen für die raumwissenschaftliche Energieforschung. Erkner. = IRS Working Paper, 50.

Moss, T.; Gudermann, R.; Röhring, A. (2009): Zur Renaissance der Gemeinschaftsgut- und Gemeinwohlforschung. In: Bernhardt, C.; Kilper, H.; Moss, T. (Hrsg.): Im Interesse des Gemeinwohls. Regionale Gemeinschaftsgüter in Geschichte, Politik und Planung. Frankfurt am Main, New York, 31-49.

Müller, J. R.; Holstenkamp, L. (2015): Zum Stand von Energiegenossenschaften in Deutschland. Aktualisierter Überblick über Zahlen und Entwicklungen zum 31.12.2014. Lüneburg. = Arbeitspapierreihe Wirtschaft \& Recht, 20.

MWE - Ministerium für Wirtschaft und Europaangelegenheiten des Landes Brandenburg (Hrsg.) (2011): Energieland Brandenburg. Newsletter - Nr. 2/2011. Potsdam.

Naumann, M.; Moss, T. (2012): Neukonfiguration regionaler Infrastrukturen. Chancen und Risiken neuer Kopplungen zwischen Energie- und Abwasserinfrastruktursystemen. Müncheberg. = ELaN Discussion Paper, 1.

Ohlhorst, D.; Schön, S. (2010): Windenergienutzung in Deutschland im dynamischen Wandel von Konfliktkonstellationen und Konflikttypen. In: Feindt, P. H.; Saretzki, T. (Hrsg.): Umwelt- und Technikkonflikte. Wiesbaden, 198-218.

Ottinger, G.; Hargrave, T.; Hopson, E. (2014): Procedural justice in wind facility siting: Recommendations for state-led siting processes. In: Energy Policy 65, 662-669.

Otto, A.; Leibenath, M. (2013): Windenergielandschaften als Konfliktfeld: Landschaftskonzepte, Argumentationsmuster und Diskurskoalitionen. In: Gailing, L.; Leibenath, M. (Hrsg.): Neue Energielandschaften. Neue Perspektiven der Landschaftsforschung. Wiesbaden, 65-75. = RaumFragen: Stadt - Region Landschaft.

Reck, H.-J. (2012): Rekommunalisierung: Modelle einer bürgernahen, nachhaltigen Energieversorgung. In: RaumPlanung 158/159, 243-248.

Saretzki, T. (2010): Umwelt- und Technikkonflikte: Theorien, Fragestellungen, Forschungsperspektiven. In: Feindt, P. H.; Saretzki, T. (Hrsg.): Umwelt- und Technikkonflikte. Wiesbaden, 33-53.

Ströbele, W.; Pfaffenberger, W.; Heuterkes, M. (2012): Energiewirtschaft. Einführung in Theorie und Politik. München.

Van der Horst, D. (2007): NIMBY or not? Exploring the relevance of location and the politics of voiced opinions in renewable energy siting controversies. In: Energy Policy 35 (5), 2705-2714.

WBGU - Wissenschaftlicher Beirat der Bundesregierung Globale Umweltveränderungen (Hrsg.) (2011): Welt im Wandel. Gesellschaftsvertrag für eine Große Transformation. Berlin.

Weiss, G. (2013): Das Klima retten - aber nicht vor der eigenen Tür? Konflikte um Anlagen zur regenerativen Energieerzeugung in Deutschland. Geographische Rundschau 65 (1), 44-49.

Wolsink, M. (2000): Wind power and the NIMBY-myth: Institutional capacity and the limited significance of public support. In: Renewable Energy 21 (1), 49-64.

Wolsink, M. (2007): Wind power implementation: The nature of public attitudes: Equity and fairness instead of ,backyard motives'. In: Renewable and Sustainable Energy Reviews 11 (6), 1188-1207.

Yildiz, Ö.; Rommel, J.; Debor, S.; Holstenkamp, L.; Mey, F.; Müller, J. R.; Radtke, J.; Rognli, J. (2015): Renewable energy cooperatives as gatekeepers or facilitators? Recent developments in Germany and a multidisciplinary research agenda. In: Energy Research and Social Science 6, 59-73.

Zografos, C.; Martínez-Alier, J. (2009): The politics of landscape value: A case study of wind farm conflict in rural Catalonia. In: Environment and Planning A 41 (7), 1726-1744. 